\title{
LES CONFLITS SOCIAUX EN AFRIQUE ANCIENNE ET LEURS RÉSOLUTIONS
}

Benjamin Diouf ${ }^{1}$

\section{Résumé}

L'Afrique ancienne a vécu des conflits sociaux, parfois violents, qui ont dressé, les uns contre les autres, les membres d'une même communauté. Mais, à chaque fois, les acteurs sociaux ont trouvé des mécanismes de résolution de ces distorsions. Existe- $\mathrm{t}$ - il une survivance de ceux-ci dans les modes de règlement de nos conflits?

\section{Mots-clés}

Conflits sociaux; mécanismes; résolution; médiation; parenté à plaisanterie; lignée; acteurs sociaux.

\section{Resumo}

A África antiga passou por conflitos sociais, às vezes violentos, que fez os membros de uma mesma comunidade se levantarem uns contra os outros. Mas a cada vez, os atores sociais encontravam mecanismos de resolução para essas distorções. Podem esses mesmos mecanismos ter permanecido nos modos de resolução de nossos conflitos?

\section{Palavras-chave}

Conflitos sociais; mecanismos; resolução; mediação; relação com a comicidade; linhagem; atores sociais.

\section{Introduction}

Au cours de l'Antiquité africaine, les sociétés, surtout celle égyptienne dont l'histoire est la mieux connue, étaient frappées par des conflits aux causes

\footnotetext{
${ }^{1}$ Assistant titulaire - Université Cheikh Anta Diop de Dakar, Dakar, Senegal. E-mail: benjdiouf067@yahoo.fr
} 
diverses. Ceux-ci avaient parfois dressé violemment, les uns contre les autres, des citoyens qui partageaient au quotidien le même pain, les mêmes lieux. L'ordre social et la paix se retrouvaient ainsi perturbés. Les peu- ples, par leurs comportements et leurs actes, retournaient à l'état animal. La violence n'épargnait personne. Dans de pareilles situations, il deve- nait impératif d'agir pour retrouver la quiétude et la cohésion sociales d'antan; ce que faisaient toujours les Anciens. Aujourd'hui encore, nous ne sommes guère étrangers à ces troubles sociaux. Notre quotidien reste toujours marqué par des conflits sociaux que nous nous évertuons à ré- gler. Mais existe-il des liens entre nos manières de résoudre nos discor- des et celles de nos ancêtres? Pour répondre à cette interrogation, nous exposerons d'abord quelques conflits sociaux anciens et modernes rela- tés par des auteurs, comme Hérodote, pour ensuite nous intéresser à leurs mécanismes de résolution.

\section{I - Quelques conflits sociaux}

Les auteurs anciens se sont beaucoup intéressés à l'Afrique et à la vie de ses hommes. Ils ont laissé à la postérité de nombreuses et précieuses informations sur la vie sociale des Egyptiens, des Ethiopiens et des Libyens. Dans leurs écrits, ils ont parfois fait écho des conflits qui ont bouleversé l'ordre social. Grâce à eux, nous connûmes qu'une vaste révolution sociale, assez brutale, secoua l'Egypte vers 2260 av. J.C. Le peuple, plongé dans une misère totale due à la chute de l'état unitaire, à la faiblesse des crues et à l'accaparement des terres par la famille royale, les gouverneurs de province et les temples, se révolta et déversa sa colère sur les riches. Les biens de ceux-ci furent pillés et les sépultures des dignitaires, remplies d'objets précieux, furent saccagées par des chercheurs de richesse. Ce triste sort, réservé aux riches, est dévoilé dans toute sa barbarie par le scribe Ipou-our qui, dans Leslamentations, écrivit: “(...) Voyez donc, les hommes démunis sont devenus propriétaires de richesses et celui qui ne pouvait faire pour lui-même une paire de sandales possède des monceaux. Voyez donc, les riches se lamentent, les miséreux sont dans la joie, et chaque ville dit: "laissez-nous chasser les puissants de chez nous." (...) Voyez donc, l'or et la lapis-lazuli, l'argent et le turquoise, la cornaline et le bronze, la pierre de Nubie entourent le cou des servantes, tandis que les nobles dames errent à travers le pays et que les maîtresses de maison d'autrefois disent: "Ah! Puissions-nous avoir quelque chose à manger!" ${ }^{2}$

\footnotetext{
${ }^{2}$ http://www.matierevolution.fr/spip.php?article222

Site web visité le 24/08/2018 à 16h 20 mn GMT.
} 
Le palais royal et les résidences luxueuses des gouverneurs des nomes furent attaqués et mis à sac par les manifestants. Le pouvoir royal échappa aux mains du pharaon et le pays sombra dans un chaos indescriptible tel que l'attestent ces propos d'un scribe, témoin de la situation: "La résidence royale a été ravagée en une heure.." ${ }^{3}$ L'historien Manéthon décrit bien cette confiscation du pouvoir par la rue en révélant qu'il y eut "soixante dix rois en soixante dix jours."

Qui plus est, le peuple égyptien vécut, durant le Nouvel Empire, un conflit social sans précédent au cours de l'Antiquité. Au XII ${ }^{\text {ème }}$ siècle avant notre ère, l'Egypte connut des difficultés politiques et économiques qui vont avoir un impact sur la vie sociale. Le pharaon Ramsès III devait faire face aux invasions libyennes, à la conspiration de Tiyi, sa seconde épouse, cherchant à l'éliminer mais surtout à la corruption qui gangrénait l'administration et appauvrissait la société. C'est dans ce contexte qu'eut lieu la grève des ouvriers de Deir el-Médineh ${ }^{5}$ qui travaillaient à la nécropole royale sise à la Vallée des Rois. Cette grève débuta le 10 du mois de Péret dans l'année 29 de Ramsès III. Celle-ci, qui n'était au commencement qu'un simple mouvement de protestation contre le retard des paiements et la qualité des produits, devint vite un conflit continu.

Etant restés sans nourriture et sans vêtements pourvus par l'état central, les ouvriers, affamés et démunis, abandonnèrent leur travail et leurs logements pour occuper des édifices publics et des temples afin d'attirer l'attention des autorités sur leur misérable sort. Ces dernières, face à la pression montante des ouvriers, leur distribuèrent quelque vivre et promirent un règlement rapide de la situation. Mais tel ne fut pas le cas et les ouvriers continuèrent à sortir dans la rue, en occupant bâtiments administratifs et lieux de culte, pour exiger le paiement de leur dû. Ce fut au total trois mouvements de grève lors desquels des autorités locales, comme le chef de police Mentmosès ou le gouverneur de Thèbes, et des prêtres, intervinrent pour calmer les ardeurs des grévistes grâce à des distributions de vivre et des paroles conciliantes.

\footnotetext{
${ }^{3}$ http://www.matierevolution.fr/spip.php?article222

Site web visité le 24/08/2018 à 16H 20 mn GMT.

${ }^{4}$ Histoire générale de l'Afrique, II Afrique ancienne, Présence Africaine/ Edicef/Unesco, 1987, p. 105.

${ }^{5}$ Les ouvriers qui travaillaient pour le Pharaon étaient payés mensuellement en rations alimentaires telles que 10 miches de pain, une mesure de bière, des légumes, des poissons et des vêtements. Certains avaient même à leur service des jardiniers. Ils étaient donc très bien entretenus par le roi. Ceux qui œuvraient à la nécropole royale pour Ramsès III, pendant la grève, peuvent être estimés à 120 ouvriers répartis en deux équipes et vivant dans plus d'une soixantaine de maisons avec leurs femmes et leurs enfants. Il y avait parmi eux des maçons, des sculpteurs, des peintres et des tailleurs de pierres.
} 
Des siècles, bien après ces différents événements, Hérodote nous apprend que les soldats égyptiens ont eu à manifester leur courroux au pharaon Psammétique ${ }^{6}$. En effet, celui - ci, comme ses prédécesseurs, avait affecté des troupes aux garnisons sises à ses frontières avec les Ethiopiens, les Arabes et les Assyriens. Mais, malheureusement, les soldats en service à ces postes restèrent longtemps, trois ans précisément, sans être relevés par d'autres. Cette longue absence, de leur patrie et de leur famille, suscita leur colère. Ils se révoltèrent et désertèrent leurs casernes. Cette révolte de l'armée égyptienne ne fut pas la seule. Il y eut le conflit qui opposa le pharaon Apriès à une partie de son armée: "Ayant envoyé contre les Cyrénéens une grande expédition, Apriès subit un grand échec; les Egyptiens lui en firent grief et se révoltèrent contre lui; ils se figuraient qu'il les avait envoyés de propos délibéré à un désastre certain, pour causer leur perte et régner lui-même avec plus de sécurité sur le reste de la population. Indignés de cela, ceux qui étaient revenus et les amis de ceux qui étaient morts se révoltèrent ouvertement."7.

En Ethiopie, la reine Candace, dans le souci de sécuriser son royaume, avait attaqué l'armée romaine en Egypte. Strabon ${ }^{8}$, qui raconte les faits, nous apprend que Candace avait profité d'une réduction du nombre des soldats romains en Egypte, dont certains étaient partis en campagne contre les Arabes, pour attaquer et prendre Syène, Eléphantine, Philae et quelques statues de César. Mais mal lui en prit puisque le chef de l'armée romaine, Pétrone qui dirigeait la campagne contre les Arabes, revint vite sur ses pas et battit les envahisseurs qu'il poursuivit jusque dans leur pays où il s'empara de la capitale Napata, sans réussir à capturer Candace, retranchée dans sa forteresse.

Enfin, l'Afrique moderne reste marquée par des conflits sociaux, aux origines parfois lointaines, qui secouent beaucoup de pays. Fatigués des humiliations,

\footnotetext{
${ }^{6}$ Hérodote, Histoires II, texte établi et traduit par Ph. E. Legrand, Paris, Les Belles Lettres, 1948, 30. Pour cette histoire, voir aussi Diodore de Sicile, Bibliothèque Historique I, section seconde, texte établi et traduit par L'abbé Terrasson, Paris, 1737, XX.

${ }^{7}$ Hérodote, Histoires II, texte établi et traduit par Ph. E. Legrand, Paris, Les Belles Lettres, 1948, 161.

Il convient de noter ici que le malaise a commencé dans l'armée égyptienne depuis qu'Apriès a intégré dans celle-ci des éléments grecs, des Cariens et des Ioniens, qu'il aurait mieux traités que les soldats égyptiens. D’ailleurs, la révolte des soldats égyptiens après la défaite contre Cyrène est due à la non participation aux combats des Grecs qui étaient dans l'armée égyptienne. Ceci fut vu par les Egyptiens comme une volonté manifeste de les éliminer. Notons également que l'incorporation de Cariens et d'Ioniens dans l'armée égyptienne se produisit, avant Apriès, sous les règnes de Psammétique et d'Amasis, selon toujours Hérodote, Histoires II, 152-154.

${ }^{8}$ Strabon, Géographie Livre XVII-1, traduction française Amédée Tardieu, Paris, Hachette, 1909, 54.
} 
des tortures et des exploitations coloniales, les peuples africains se résolurent à jeter leurs dernières forces dans des combats libérateurs. Ceux-ci furent incités et dirigés par l'élite intellectuelle africaine formée à l'école occidentale. En effet, les intellectuels noirs, outrés par les maltraitances et la misère de leurs frères de race, avaient provoqué l'éveil de conscience du peuple résigné à la soumission. Ils avaient fait entrevoir à la masse populaire sa capacité à se libérer du joug occidental pour vivre des lendemains meilleurs. Le seul espoir de liberté et de bien-être social, que les intellectuels faisaient miroiter aux yeux du peuple, suffit à fédérer toutes les forces vives des nations colonisées. La notion d'indépendance devint subitement synonyme d'une vie "paradisiaque". Les colonisés engagèrent et gagnèrent les luttes pour les indépendances. Les premières années des indépendances maintinrent toujours l'espoir d'une vie meilleure même si les attentes ne furent pas encore satisfaites.

Cependant, une dizaine d'années après les indépendances, un désenchantement total gagna les peuples africains déçus de la gouvernance et des promesses non tenues de leurs frères de race à la tête des pays. Les années, qui vont suivre, vont ouvrir une page sombre de l'histoire de l'Afrique moderne marquée par des crises sociopolitiques de grande envergure. La famine et les conflits fonciers ou interethniques donnent un visage désolant de cette Afrique nouvelle. En Angola, au Libéria, au Soudan, pour ne citer que ces pays, c'était partout l'horreur. Jusqu'en ce 21ème siècle encore les crises sociales secouent quelques pays africains et font des milliers de morts. Ces conflits africains ont les mêmes causes que ceux jadis connus en Egypte pharaonique. Il s'agit de la mal gouvernance, de l'accaparement des terres et des richesses par les dirigeants et de l'injustice qui frappent certaines couches sociales. Il urge donc d'apporter des solutions à ces maux, qui déchirent l'Afrique, mais que celles-ci soient surtout adaptées au contexte africain, comme le souhaite Sam G. Amoo: "Une compréhension conceptuelle des origines et de la dynamique des conflits africains est une condition préalable à l'élaboration et à l'application de concepts et de stratégies réalistes et pertinents à prévenir, à gérer ou à régler les conflits."(Amoo 1997: 02). Une solution africaine aux problèmes du continent est donc envisageable. Dans les pages qui vont suivre, nous découvrirons certains de ces mécanismes de résolution de conflits hérités du passé africain.

\section{II - Les mécanismes de résolution des conflits}

Parmi les moyens de résolution des conflits en Afrique ancienne, nous avons la médiation. Celle-ci intervient lorsque les parties opposées ne parviennent pas à entrer en contact ou quand elles sont incapables de résoudre elles-mêmes leurs différends. C'est dans ces circonstances qu'intervient une tierce per- 
sonne ou un groupe d'individus, pouvant même être partie prenante au conflit, soucieux de restaurer les liens brisés par le conflit. Nous estimons que la médiation a été utilisée en Egypte ancienne, à la fin de la Première période intermédiaire (-2280/-2060), pour ressouder les liens sociaux. Le roi Mentouhotep II avait réussi à mater tous les fauteurs de trouble et à faire régner l'ordre. Toutefois, il restait à rétablir l'entente entre des citoyens qui s'étaient dressés les uns contre les autres. Après les troubles, les Egyptiens se regardaient encore en chien de faïence car les esprits restaient toujours traumatisés par les scènes de violence de la veille. Il fallait donc pacifier les cœurs et cela ne pouvait se faire par la force. Seul le peuple, d'un commun accord, avait le pouvoir de panser ses blessures internes. Il était nécessaire de s'assoir ensemble pour discuter et s'entendre sur un nouvel ordre social; ce qui se fit, car les Egyptiens dressèrent un ensemble de valeurs morales garantissant l'épanouissement de chaque individu. Le papyrus de Protestations du paysan éloquent ${ }^{9}$ confirme bien ce nouvel ordre social qui s'est dessiné avec le concours de toutes les couches sociales.

La médiation a aussi montré ses bienfaits pendant la grève des ouvriers de Deir el-Médineh. Affamés et laissés à eux-mêmes, les ouvriers avaient cessé toute activité pour monter leur courroux à leurs supérieurs. Ils quittèrent à plusieurs reprises leurs résidences et occupèrent des édifices publics et des temples. Mais, à chaque fois l'intervention d'une tierce autorité, comme le gouverneur de Thèbes, ramena le calme. Pourtant, tous les germes d'une révolte meurtrière étaient réunis. Les ouvriers et leurs familles avaient faim et leurs rations alimentaires tardaient toujours ainsi que l'illustre ce passage extrait du Papyrus de la grève et repris par Nelson Pierrotti: “(...) Nous en sommes arrivés là, à cause de la faim et de la soif, par le manque de vêtements, de poisson et de légumes. Ecris à notre seigneur, le Pharaon, à ce sujet, écris aussi au gouverneur, qui est notre supérieur. Faites-le pour que nous puissions vivre!" (Pierrotti 2009: 06). Ces propos des grévistes, adressés aux fonctionnaires du temple funéraire de Ramsès II, montrent combien les Egyptiens plaçaient leur foi dans la médiation pour trouver un dénouement heureux à une crise. La foule des ouvriers a été affamée par des fonctionnaires véreux qui détournaient les rations et dont ils pouvaient bien saccager les maisons pour se nourrir. Mais les grévistes ont sollicité la médiation de leur supérieur immédiat, le gouverneur, pour un retour à la normale de leur ravitaillement. Leur espoir ne fut pas vain puisque l'intervention de celui-ci et d'autres fonctionnaires leur a permis à chaque fois de recevoir une partie de leurdû.

\footnotetext{
${ }^{9}$ Ce document, daté de la Xème dynastie, raconte l'histoire d'un paysan qui fait valoir ses droits devant un riche qui lui a arraché ses biens; ce qui atteste de l'égalité retrouvée ensemble par les Egyptiens.
} 
Lors des crises égyptiennes, relatées par Hérodote, nous avons toujours noté cette volonté affichée de retrouver la paix grâce à la médiation. Le roi Psammétique en a donné l'exemple. Face à la défection d'une partie de son armée, il n'a pas cherché à user de la force pour rappeler les soldats à l'ordre. Il privilégia la négociation et envoya, comme médiateurs, quelques - uns de ses hommes de confiance pour raisonner les mutins. Même lorsque ceux-ci restèrent figés sur leur position, Psammétique poursuivit ses efforts de paix et les rencontra pour leur faire changer d'avis sans pour autant y parvenir. Cette tactique de ce pharaon, même si elle ne fut pas payante, montre que la médiation est la meilleure manière de rétablir la paix. De même, la guerre meurtrière qui opposa Candace à l'armée romaine eut un dénouement heureux grâce à une ambassade que la reine avait envoyée à César.

Cependant, aussi utile que soit la médiation dans le règlement d'un conflit, il faut souligner qu'elle ne peut être menée par n'importe qui. L'échec de la médiation que le pharaon Apriès confia à son lieutenant Amasis, suite à la révolte d'une partie de ses soldats, en est un exemple. Apriès a échoué dans sa tentative parce que son envoyé n'était pas neutre, comme l'indique ce passage: "Instruit de l'événement, Apriès envoya vers les révoltés Amasis pour les apaiser en leur parlant. Amasis se rendit près d'eux; il s'efforça de retenir les Egyptiens et de les empêcher de s'insurger; mais, tandis qu'il parlait, un des Egyptiens qui était debout derrière lui le coiffa d'un casque, et déclara qu'en le coiffant ainsi il le désignait pour être roi. Cela, je pense, ne se passait pas contre le gré d'Amasis; sa conduite le montra; car, après que les Egyptiens révoltés l'eurent fait roi, il prit des dispositions pour marcher contre Apriès." ${ }^{10}$ Amasis, envieux du pouvoir de son roi, avait retourné la situation en sa faveur. Ce fait illustre qu'un médiateur doit être neutre, sage, pondéré et connaître les us et les coutumes. C'est pourquoi, dans toutes les sociétés traditionnelles africaines, la médiation était assurée par les sages que sont les vieilles personnes. Celles-ci parvenaient toujours à concilier, sous l'arbre à palabre, les parties opposées grâce aux différentes qualités dont nous avons fait état. Grâce à leur tact, les médiateurs faisaient sentir aux parties en conflit que l'arbre à palabre n'est pas un lieu de jugement, mais d'éradication de la violence et d'établissement de la paix. Ils ne faisaient éprouver à personne le poids de son tort, car tout se faisait dans la convivialité et chaque partie en sortait gagnante.

En outre, les femmes ne restèrent pas les bras croisés face aux conflits qui secouaient leurs sociétés. Elles ont toujours joué un rôle dans la gestion et la défense des cités. En Egypte, le relief de Deshasheh, qui date de l'époque

${ }^{10}$ Hérodote, Histoires II, 162; voir aussi Diodore de Sicile, Bibliothèque historique I, section seconde, texte établi et traduit par L'abbé Terrasson, Paris, 1737, XXI. 
héracléopolitaine, montre des femmes défendant vaillamment une ville assiégée par l'ennemi. On se rappellera aussi des reines qui ont eu à gouverner l'Egypte pharaonique, comme Hatshepsout durant le Nouvel Empire. Celle-ci, qui appartient à la XVIII ${ }^{\text {ème }}$ dynastie, a marqué l'esprit des Egyptiens tout au long de ses vingt ans de règne grâce au développement de l'économie du pays. Mais le plus remarquable fut la paix qu'elle a su maintenir tout au long de cette période alors que l'Egypte portait encore les stigmates de la lutte de libération contre les Hyksos. Rappelons-le, ceux-ci furent chassés d'Egypte

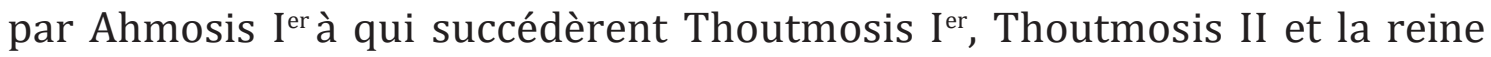
Hatshepsout. Pendant le règne de cette dernière, les germes d'une frustration ne manquèrent certes pas en Egypte, mais la reine a su se concilier tout le peuple pour réaliser ses grands chantiers, comme le temple de Thèbes. Hérodote ${ }^{11}$ signale également, en Libye, des femmes zauèces qui conduisent des chars.

En Ethiopie ancienne, nous retrouvons toujours ce rôle important que jouaient les femmes dans le règlement des conflits sociaux. Dans cette contrée vivait une ethnie nomade, celle des Troglodytes Mégabares. Ceux-ci étaient des pasteurs qui se déplaçaient constamment à la recherche de nouvelles terres pourvoyeuses de nourriture pour leurs bêtes. Au cours de leurs transhumances, il arrivait que deux clans, de cette même ethnie, se retrouvassent sur un même pâturage qu'aucun ne voulait céder à l'autre. Alors, il s'engageait une guerre meurtrière dont l'issue pacifique venait de l'intervention des femmes âgées: "Ces combats sont terminés par de vieilles femmes qui se jettent au milieu de la mêlée, et qui sont fort respectées. Il n'est permis à personne de les frapper de quelque manière que ce soit. Aussi, dès qu'elles paraissent, on cesse de tirer." ${ }^{\prime 2}$

Tous ces faits montrent que les femmes s'investissaient pleinement pour le développement, la sécurité et la paix dans leurs sociétés. Une résolution d'un conflit entreprise par des femmes avait beaucoup de chance de succès. Ce fait s'explique par l'idée que l'Africain avait de la femme. Celle-ci, même étant une épouse, était toujours perçue comme une mère. Ainsi on lui devait respect et considération et sa voix était bien écoutée et prise en compte dans les décisions surtout lorsqu'il s'agissait d'une femme d'un certain âge.

Aujourd'hui, les femmes africaines sont des actrices importantes dans la résolution des conflits sur le continent grâce à leurs médiations. Elles ont permis de sceller la paix définitive lors du conflit meurtrier qui décimait les po-

\footnotetext{
${ }^{11}$ Hérodote, Histoires $I V$, texte établi et traduit par Ph. E. Legrand, Paris, Les Belles Lettres, 1960, 193-194.

${ }^{12}$ Diodore de Sicile, Bibliothèque Historique III, texte établi et traduit par M. Ferd Hoefer, Paris, Charpentier, 1846, XXXIII.
} 
pulations du Libéria et de la Sierra Léone. Ce fut le Réseau des Femmes du fleuve Mano pour la paix qui a ouvert la voie aux négociations des chefs d'état qui se sont soldées par un accord de paix à Rabat sous l'égide de la CEDEAO et de l'U.A. L'action des femmes est également remarquable dans la recherche de la paix en Casamance, au Sénégal, où les USANA ${ }^{13}$ se sont fait distinguer. Avec les prêtresses à leur tête, les USANA célèbrent des rituels de délivrance pour les combattants du MFDC (Mouvement des Forces Démocratiques de la Casamance) en vue de les délivrer des pactes et des engagements qu'ils ont contractés en entrant dans le conflit.

Ces associations mènent également des campagnes de sensibilisation auprès des populations, des rebelles et des autorités sénégalaises sur les conséquences désastreuses de la guerre. Elles sont très écoutées grâce à leur pouvoir mystique et à leur genre. Personne, plus que ces femmes, ne ressent les méfaits du conflit. Elles sont mères et épouses des belligérants; ce qui motive leur appel à la paix. Cela a fait que depuis les années 2000, les représentantes des USANA œuvrent au quotidien au retour de la paix et sont parfois invitées aux négociations entre le MFDC et l'Etat sénégalais lorsqu'elles n'en sont pas les initiatrices.

L'action actuelle des femmes africaines dans la recherche et la consolidation de la paix constitue un héritage ancestral. Cela est bien perçu et mis en exergue par Djibril Tamsir Niane qui, au sujet de l'implication des femmes africaines dans la résolution des conflits, déclare: "Il y a tout simplement qu'avec l'entrée en scène des femmes, une valeur africaine fondamentale entrait en jeu: à savoir le respect quasi religieux que l'Africain voue à la femme, à la mère, celle qui donne la vie." ${ }^{4}$

Tel était, dans le passé, la médiation qu'assurent actuellement la société civile, des personnalités politiques ou non, des organisations et des ONG, dans l'esprit hérité de nos Ancêtres.

\footnotetext{
${ }^{13}$ On appelle l'Association USANA parce que celle-ci s'est réunie pour la première fois sous un fromager, arbre appelé usana chez les Diola. La première de ces associations rituelles urbaines fut créée par Marie Afinkoh dans le quartier de Nyéfoulène dans les années 1930-1940. Depuis lors, il existe une liste incomplète d'une dizaine d'USANA déclarées à la mairie de Ziguinchor. Ces associations, créées par des femmes âgées aux pouvoirs "mystiques" reconnus et autour desquelles se forme un chapitre de suivantes, interviennent en cas de malheur privé ou public en organisant des sacrifices et des cérémonies de prière.

${ }^{14}$ Djibril Tamsir Niane "La parenté à plaisanterie: origine historique, fonction préventive et régulatrice dans l'espace ouest - africain." in "Initiative de valorisation des capacités africaines endogènes dans la gouvernance et la prévention des conflits.", Conakry (Guinée), du 9 au 11 mars 2005, p. 9.
} 
Mieux encore, la parenté à plaisanterie ${ }^{15}$ est présentement l'un des mécanismes employé pour restaurer la paix sociale dans certaines zones en conflit sur le continent noir. Il est difficile de situer son origine. Toutefois, l'objectif visé par la parenté à plaisanterie, à savoir favoriser et maintenir une harmonie sociale parfaite et durable, nous permet de la rapprocher du principal fondement de la société égyptienne ancienne. En effet, le pilier de l'égalité et de la cohésion sociale, pour les Egyptiens, est la maât. Maât, dans la mythologie égyptienne, est la déesse de l'ordre, de l'équilibre du monde, de l'équité, de la paix, de la vérité et de la justice. L'attachement des Egyptiens à cette divinité a développé chez eux un mode de conduite et de vie que nous appelons la maât et qui renvoie à l'ensemble des conditions qui font apparaître et qui renouvellent la vie. Cette maât, chez les Egyptiens, s'oppose à tout trouble politique et social qui perturberait le mouvement des astres, l'équilibre de l'univers, mettant ainsi en danger les vies humaines, car l'ordre social, voire la vie humaine, et la prospérité sont tributaires de l'ordre cosmique. Le garant de cette harmonie sur terre est le roi, représentant de la déesse Maât. C'est ce qui confère au dirigeant la notion de justice, symbole d'une bonne gouvernance.

La maât est une philosophie de la vie commune basée sur une justice qui inspire le politique et comprend toutes les règles qui gouvernent la cité. Ce visage de la maât égyptienne dont se pare le roi est bien visible dans la désignation du pouvoir chez les Seerer siin. Chez ceux-ci, on emploie le mot maât pour désigner le pouvoir politique dans le sens d'une gouvernance fondée sur la justice et l'harmonie sociale. En revanche, tout pouvoir fondé sur l'injustice et la violence est appelé maât manta. Et plus intéressant encore, cette même ethnie se sert du mot maas, ayant le même radical que maât, pour désigner la parenté à plaisanterie. Tous ces deux mots évoquent, dans nos souvenirs, la déesse égyptienne Maât et ont un même objectif. La pratique de la maât, chez les Egyptiens, visait à respecter les règles traditionnelles et générales de la solidarité d'action et de communication; ce que cherche à réaliser et à consolider le maas. Celui-ci crée toutes les conditions de la réalisation de cette union des cœurs. Un maasiir (parent à plaisanterie) s'abstient de faire du mal à l'autre en paroles et en actes et reste attaché à lui dans l'épreuve. N'est-ce pas ce dont se réjouit le défunt au chapitre 125 du Livre des morts, lorsqu'il déclare "ne pas avoir blessé ni affligé, ne pas avoir nui...", pour montrer son application de la maât? In fine, on pourrait donc bien admettre une origine pharaoni-

\footnotetext{
${ }^{15}$ Pour mieux faire comprendre la notion de parenté à plaisanterie, nous renvoyons nos lecteurs à cette définition de Raphael Ndiaye, extraite de Pluralité ethnique, convergences culturelles et citoyenneté en Afrique de l'Ouest, Enda Tiers-Monde, 2004, p. 29: "C'est un ensemble de liens conviviaux, privilégiés établis par l'Ancêtre, activés dans une démarche personnelle renouvelée et qui fonctionne sur la base de l'humour et la dérision courtoise."
} 
que de la parenté à plaisanterie. Cette probabilité demeure renforcée par les liens de parenté établis par Cheikh Anta Diop ${ }^{16}$ entre les Seerer siin et les Egyptiens anciens.

Par ailleurs, nous pouvons noter que la parenté à plaisanterie, bien connue et pratiquée par beaucoup d'ethnies africaines telles que les Seerer, les Peulhs, les Bambaras ou les Wolofs, a laissé des traces de son institution au Mali durant le règne de Soundiata Keita. Celui-ci, après sa victoire sur le roi sosso Soumagourou Kanté, avait jugé nécessaire d'instaurer une paix durable dans tout son empire et un code de conduite où l'être humain trouverait son plein épanouissement. C'est dans cette optique qu'il convoqua en 1236, à Kouroukan Fouga, l'assemblée des peuples sous sa domination. Cette grande rencontre permit d'établir consensuellement la Charte du Mandé que beaucoup d'historiens considèrent, aujourd'hui, comme la première déclaration des droits de l'homme de l'histoire humaine. Pour lubrifier davantage les relations interethniques et prévenir d'autres conflits, l'assemblée, sous la houlette de Soundiata, institutionnalisa la parenté à plaisanterie.

Dans un passé fort récent, la parenté à plaisanterie a contribué à réconcilier Maliens et Voltaiques (Burkinabais actuels) lors de la guerre qui les opposa en 1970, selon ces propos: "Le Président Sékou Touré invita les Présidents Moussa Traoré et Sangoulé Lamizana à une conférence à Conakry; l'illustre griot Sory Kandia Kouyaté dans une belle évocation du passé rappela le pacte d'amitié entre Samogo et Bambara, clans des Présidents Lamizana et Moussa Traoré. Le rappel du pacte ancestral eut le don de calmer le courroux guerrier des deux chefs qui s'embrassèrent devant la foule médusée. Ainsi fut enterrée la hache de guerre." (Niane 2005: 10).

Présentement, la parenté à plaisanterie est le principal levier sur lequel s'appuie l'Association culturelle Ageen et Diambogne ${ }^{17}$ pour un retour définitif de la paix en Casamance, au sud du Sénégal. Celle-ci, basée sur la parenté historique entre Seerer et Diola, a même associé l'ethnie peulh, qui a des liens anciens avec les Seerer, à la recherche de la paix. Ses membres exploitent les liens sanguins fraternels qui unissent ces ethnies pour en faire les fers de lance

\footnotetext{
${ }^{16}$ Sur ce point, voir Cheikh Anta Diop (1954: 388;391).

${ }^{17}$ Ageen et Diambogne est une association culturelle créée en 1994. Selon la tradition, Ageen et Diambogne sont deux sœurs jumelles. Un jour, leur maman les avait envoyées chercher du bois de chauffage. Les deux sœurs furent surprises au retour par une forte tempête qui fracassa leur pirogue en deux parties. Ageen s'accrocha sur l'une et fit entraînée par le vent jusqu'à Kalobane en Casamance où elle vécut et donna naissance aux Diola, tandis que Diambogne échoua, sur l'autre partie de la pirogue, à Diakhanor dans le Sine où elle engendra les Seerer. Ce qui atteste le cousinage entre Diola et Seerer et fonde leur parenté à plaisanterie.
} 
de la quête de la paix dans cette partie sud du Sénégal. La possibilité qu'offre la parenté à plaisanterie à pouvoir se mouvoir chez l'autre sans être inquiété et à lui parler franchement, dans un climat convivial et humoristique, est très importante pour la réconciliation des parties opposées.

En effet, chacun des parents à plaisanterie reste conscient des liens sanguins ancestraux qui l'unissent à l'autre et s'efforce de satisfaire les désirs de celui-ci. La trahison ou le non respect de la parole donnée sont lourds de conséquence pour son auteur qui ne tarde pas à subir la punition de ses ancêtres. C'est ce qui favorise le succès de la parenté à plaisanterie lors des négociations et explique son usage par beaucoup d'ethnies en Afrique au sud du Sahara où elle contribue à instaurer la paix et à renforcer la cohésion sociale. Par exemple, elle a permis l'accalmie dans le conflit casamançais en offrant un espace de dialogue aux représentants de l'Etat sénégalais et du MFDC à Foundiougne en février 2005.

Enfin, il est important de noter que les textes anciens ne rendent pas comptent de tous les mécanismes qu'utilisaient les Africains pour restaurer la paix sociale. Nos auteurs nous ont juste fait part de l'issue de quelques conflits ayant attiré leur attention car ayant mis aux prises des rois ou des sujets contre leurs dirigeants. N'y eut-il pas des conflits entre citoyens ou familles? Les auteurs anciens les ont passés sous silence; ce qui ne vaut nullement dire qu'il y'en avait pas. Il y eut bien des conflits parfois violents entre les habitants, et nous pouvons connaître leur mode de résolution en examinant l'organisation et le fonctionnement de nos sociétés traditionnelles. Dans celles-ci, chaque lignée a un chef, qui est le plus âgé, à qui tous les autres membres de la famille vouent un respect quasi religieux. C'est celui-ci qui recevait les plaintes des autres et intervenait lorsqu'il y avait la violence, ou des faits pouvant la provoquer, au sein de la famille. Il convoquait dans sa case les parties en conflit, leur rappelait leurs liens familiaux, leurs droits et devoirs mutuels et usait avec tact de toute sa personnalité pour les réconcilier et obtenir d'elles la promesse d'une paix définitive. Avant de prendre congé de leur hôte, les deux parties partageaient un repas ou une boisson dans une ambiance fraternelle.

Quand le conflit opposait des personnes ou des familles différentes, ce sont ces mêmes chefs de lignée qui ramenaient la paix. Le plus jeune d'entre eux effectuait le déplacement chez son aîné pour discuter et trouver ensemble une solution dans l'intérêt des deux familles. Toutefois, il pouvait arriver que la gravité des faits ayant engendré le conflit ou l'impartialité des chefs de lignée fût un obstacle à cette rencontre. Dans ce cas précis, un chef d'une autre lignée amie jouait les bons offices. C'est ainsi, par exemple, que se réglaient les conflits chez les Seerer siin, une ethnie du Sénégal, où l'on recourt encore à ce mécanisme lorsqu'il y'a problème ou conflit. 


\section{Conclusion}

Les conflits sont inhérents à la vie des sociétés. En effet, le choc des ambitions, l'égoïsme, la mal gouvernance, l'injustice et tant d'autres faits provoquent des frustrations qui se transforment souvent en révoltes parfois violentes. Celles-ci brisent la cohésion sociale et dressent les hommes les uns contre les autres. Toutes les sociétés ont connu et continuent à connaître ces distorsions sociales. Mais, à chaque fois, les peuples ont su se ressaisir et trouver les moyens de les résoudre. En Afrique ancienne, nous avons vu certains de ces mécanismes de résolution des crises qui peuvent être adoptés dans le règlement des conflits sociaux que nous vivons actuellement. En réveillant cet héritage endormi en chacun de nous, nous pouvons facilement rétablir la paix dans les coins troubles de nos sociétés.

\section{Sources}

Diodore de Sicile. Bibliothèque Historique I, section seconde. Texte établi et traduit par L'abbé Terrasson: Paris, 1737.

Diodore de Sicile. Bibliothèque Historique III. Texte établi et traduit par M. Ferd Hoefer, Paris: Charpentier, 1846.

Hérodote. Histoires II. Texte établi et traduit par Ph. E. Legrand. Paris: Les Belles Lettres, 1948.

Hérodote. Histoires IV. Texte établi et traduit par Ph. E. Legrand. Paris: Les Belles Lettres, 1960.

Strabon. Géographie Livre XVII-1. Traduction française Amédée Tardieu. Paris: Hachette, 1909.

\section{Références bibliographiques}

AM00, Sam G. Le défi de l'ethnicité et des conflits. Nécessitéd'un nouveau modèle, Division des interventions d'urgence. New York: PNUD, 1997.

DIOP, Cheikh Anta. Nations nègres et culture. Paris: Présence Africaine, 1954.

Histoire générale de l’Afrique, II Afrique ancienne. Présence Africaine/ Edicef/Unesco, 1987.

NIANE, Djibril Tamsir "La parenté à plaisanterie: origine historique, fonction préventive et régulatrice dans l'espace ouest - africain." in "Initiative de valo- 
risation des capacités africaines endogènes dans la gouvernance et la prévention des conflits.", Conakry (Guinée), du 9 au 11 mars 2005.

NDIAYE, Raphael, Pluralité ethnique, convergences culturelles et citoyenneté en Afrique de l'Ouest, Enda Tiers-Monde, 2004

PIERROTTI, Nelson “La première grève connue de l'histoire, XII ${ }^{\text {ème }}$ siècle, 1166 av. J.C", 2009.

\section{Site Web}

http://www.matierevolution.fr/spip.php?article222 